\title{
A DIAGNOSTIC SYSTEM FOR THE MICROGRAVITY EXPERIMENTS ON MARANGONI DROP MIGRATIONS
}

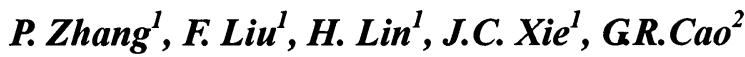 \\ 1. National Microgravity Laboratory, Institute of Mechanics, Chinese Academy of Science \\ Beijing 100080,China. Email: zp@imech.ac.cn \\ 2.Optical department, Information Engineering Institute, Beijing Institute of Technology \\ Beijing 100081, China. \\ Corresponding author Zhang Pu
}

\begin{abstract}
An optical diagnostic system designed for the microgravity experiments on Marangoni drop migrations has been depicted in the present paper. One part of the optical system was used to image and record the drops tracks; the other part was an equal-thick interferential system, it has the ability to observe the fine structures of the drop migrations. Some ground-based experiments had been performed and the results were simply discussed in the present paper.
\end{abstract}

Keywords: Fluid mechanics, Marangoni drop migrations, optical diagnostics,

space experimental device

\section{Introduction}

The Marangoni drop migration, which is the motion when the drop is in the uneven fields of temperature or concentration, is a classical question of fluid mechanics. [1] In the present paper only the migration driven by temperature gradient is discussed. When the bubbles and drops are submerged in an immiscible liquid, it will produce thermocapillary migrations driven by interfacial tension due to the temperature gradient on their surface. The effect of capillary tension is coupled with the buoyancy that makes it difficult to research the pure capillary process on the ground. While in microgravity environments, the buoyancy can be neglected and the thermocapillary migrations turn to be the dominating process. The space experiments in sustaining microgravity environments are the best chance to study the Marangoni drop migrations.

The Marangoni drop migration is important in both theory and applications. During the process of producing alloy and welding materials in space, many droplets and bubbles will occur inside the continued liquid. When the temperature field is not uniform, the Marangoni migrations will occur due to the gradient of the surface tension. The bubbles and droplets suspending in the melting liquid can be driven out by controlling the temperature. Results of those experiments will help to improve the producing process both in space and on the ground.

P. Zhang ${ }^{1}$, F. Liu ${ }^{1}$, H. Lin ${ }^{1}$, J.C. Xie ${ }^{1}$, G.R.Cao ${ }^{2}$

1. National Microgravity Laboratory, Institute of Mechanics, Chinese Academy of Science

Beijing 100080, China.

2.Optical department, Information Engineering Institute, Beijing Institute of Technology

Beijing 100081,China.

Email address: zp@imech.ac.cn 
Many experiments have been performed on the ground and the results showed the coupled migration effects of thermocapillary tension and buoyancy. [2]-[4] The recently successful space experiments on bubbles and drops migration were carried out aboard the IML-2 mission of the Space Shuttle. [5] The experiment apparatus was named BDPU (bubble, drop, particle, unit)which was provided by ESA . The apparatus consists of the systems of power, optical diagnostics, illumination and imaging. The optical diagnostics system includes a video camera, a motion picture camera and a point diffraction interferometer (PDI), the PDI can be used only in a small temperature range because of the influence of the gradient of the temperature-refractive index. This was the only apparatus which including an interferometer in so many space experiment facilities I know until now. In this experiment, the continuous liquid was 50cst silicone oil and drop liquid was Fluorinert FC-75. The diameters of the drops varied from $2.0 \mathrm{~mm}$ to $14.4 \mathrm{~mm}$, and those of bubbles ranged from $1.2 \mathrm{~mm}$ to $14.8 \mathrm{~mm}$ and the temperature gradient was $1.0 \mathrm{~K} / \mathrm{mm}$.

In 1996, by using the same apparatus BDPU, the drop migration experiments were performed aboard the LMS mission of the Space Shuttle of NASA [6]. Air and Fluorinert FC-75 were used for the bubble and drop phases, respectively, 10cst silicon oil was employed for the continuous phase. Results were found to be generally consistent with that of IML-2.

There are two important dimensionless parameters in study the nonlinear process. The influence of inertia term and heat convection should be included in this case.

$$
\begin{array}{cc}
\text { Interfacial tension Reynolds numbers } & \mathrm{Re}=\frac{V_{0} R}{v_{i}}, \\
\text { Marangoni numbers } & M_{a}=\frac{V_{0} R}{\kappa_{i}} .
\end{array}
$$

Here, the reference velocity is defined as $V_{0}=-\frac{\sigma_{T}^{\prime} R\left|\nabla T_{\infty}\right|}{\mu_{i}}, \mathrm{R}$ is the radius of the drop, $\sigma_{T}^{\prime}$, the rate of change in interfacial tension with temperature, $\nabla T_{\infty}$, the temperature gradient imposed on the continuous phase fluid, and $v_{i}, \mu_{i}, \kappa_{i}$, the kinematics viscosity, dynamic viscosity and thermal diffusivity. The subscripts $i=1,2$ stand for drop and continuous phase respectively. By formula (1), the large Reynolds numbers imply that the influence of the inertia is relatively strong and thus requiring that the drop sizes should be larger, the temperature difference be higher and viscosity be lower.

\section{Design of the space experiment}

The experiment is one of the spaceship projects of China and will be performed in the not long future. The main purpose of the experiments is to study the thermocapillary migrations of single drop with different sizes and different temperature gradients. The chief task is to observe the track of the migrations and measure the migrations velocity. The duration of the space experiments is about $90 \mathrm{mins}, 60 \mathrm{mins}$ for establishing the temperature field and $30 \mathrm{mins}$ for performing the experiments. The experiment apparatus is designed to auto-controlled by the embedded program. At the same time, it has the function of telescience. The Principle Investigates on ground can control and intervene in the space experiments through telecommands in real time.

According the experience we selected 5cst silicon oil and FC-75 as the continuous and drop liquids respectively. The experiment mediums have many advantages: the 5cst silicon oil has lower viscosity that easy to obtain larger migrations velocity, the rate of interface tension with temperature is high to obtain large Reynolds numbers. Due to the space limitation, we selected the drop sizes as $D=2 \sim 10 \mathrm{~mm}$, the temperature gradient as $\left|\nabla T_{\infty}\right|=1 \sim 1.5 \mathrm{~K} / \mathrm{mm}$. Compared with IML-2, we can 
obtain larger Reynolds numbers for the higher temperature gradient and lower liquid viscosity in the space experiments.

According to the results of numerical simulation, the trajectory of drop migrations in large Reynolds numbers would be oscillated. An optical interferential system is especially designed to observe the fine structure in its wake.

The device consists of test cell, injecting system, optical diagnostic system, image system and electrical-control system. The test cell is the core of the device, and it is the site for drop migration. The injecting system is responsible for injecting different size drops to the test cell according to the time sequent demands. The optical diagnostic system can observe the background temperature field, fluid flow field and the interference field. The image and recording system collects and records the flow field and interference images on two VTRs in real time, respectively. The electrical control system has the function of carrying out the experiments according to the program sequence, storing the scientific data and communicating with ground station [7]. Only the test cell and optical diagnostic system are introduced in the following section.

\section{Optical diagnostic system}

\subsection{Test cell}

The four $8 \mathrm{~mm}$-thick glass walls of the rectangular test cell are stick together by glue, and the interior is $40 \times 30 \times 42 \mathrm{~mm}^{3}$ (length $\times$ width $\times$ height). The former experiments showed that the time it takes to set up steady temperature field was directly proportional to the square of the height of the test cell, so the height of the test cell is selected as $42 \mathrm{~mm}$. Two Aluminum boards in the height dimension bound the cell, and an electro thermal film is mounted on the top surface to establish the temperature field. There are six thermocouples to measure the temperature in the test cell. Two on top and bottom surfaces and four on one not-pass-light side glass wall with the interval $8 \mathrm{~mm}$. Special pastern to preventing the leakage of the liquid inseparably glues the inlets of the four thermocouples across the glass wall. There is also a flexible vessel linking to the test cell to compensating the pressure in the cell.

The test cell is the key component of the whole experimental device. It was not only the site for drop migration but also the part of interferential system. In order to improve the stability, the equal-thick interferometer without maladjustment is composed of the front and rear surfaces of the test cell.

During the space experiments, the temperature data can be down linked to the application center on ground for display and storage. The scientist can judge the temperature difference in the test cell according to the temperature curves. The test cell is shown in Fig1.

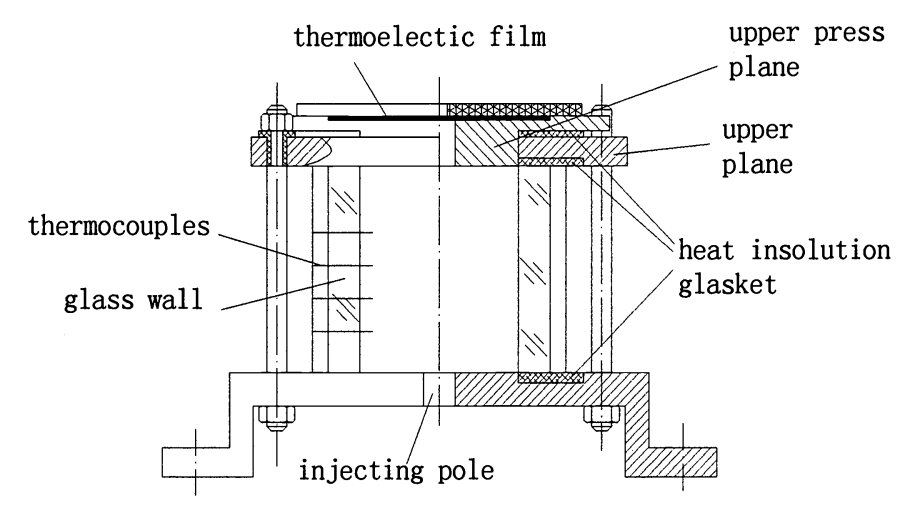

Fig.1. Sketch of Test Cell 


\subsection{Optical diagnostic system}

The optical system consists of two parts: drop track camera system and interferential system.

The CCD is the image receiver of the drop track camera system. Four LEDs are used for illuminating from the backside of the test cell, the field of vision is $56 \mathrm{~mm} \times 40 \mathrm{~mm}$, the object resolving power is $0.1 \mathrm{~mm}$ and the object depth of field is $\pm 2 \mathrm{~mm}$. The cross section of the test cell is $40 \mathrm{~mm} \times 30 \mathrm{~mm}$ and the drop diameters are varied from $2 \mathrm{~mm}$ to $10 \mathrm{~mm}$, the system can clearly image and track the drops in the whole angular view. In order to improve the efficiency of the device and obtain more related information in an experiment, the track system is required to work with the interferential system independently and simultaneously. The optical axis of the track system is deflexed $5^{\circ} \mathrm{C}$ from the other system to prevent the laser beam from producing a bright facula at the image plane center of the track system. The drop migration velocity could be obtained according the tracks of the drop migrations.

The interferential system detects the change of the interferential stripes produced by drop migrations. The illumination source is a laser $(\lambda=650 \mathrm{~nm})$, and the image receiver is another CCD. Due to the stability request of space experiments, it is designed as an interferometer without maladjustment. The equal-thick interferential field is made up of two beams reflecting from the front and the rear surfaces of the test cell. The beam reflecting from the rear surface passes through the liquid twice, so it has the information of refractive index distribution related to the temperature field of the liquid. The flow by the drop migrations could induce the variation of the temperature field around it. So it can indirectly measure the wake's fine structures through the change of the interferential stripes due to the variations of the temperature field of the experimental fluid.

Because the refractive index of the experimental liquid will vary greatly with temperature variation, (that is, the coefficient of the temperature refractive index is large), good stripes contrast can be ensured only with appropriate temperature difference. The refractive index of the liquid will vary so much for $45^{\circ} \mathrm{C}$ temperature difference and produce such an equivalent optical wedge that the stripes are too close to read. We designed the test cell in a shape of broad top and narrow bottom to compensate the influence of the temperature. The value of the wedge angle is determined by the temperature difference and the coefficient of temperature refractive index. So the interferential system is effective only in such temperature range that $45^{\circ} \mathrm{C} \pm 1^{\circ} \mathrm{C}$. The sketch of the optical system is shown in Fig2.

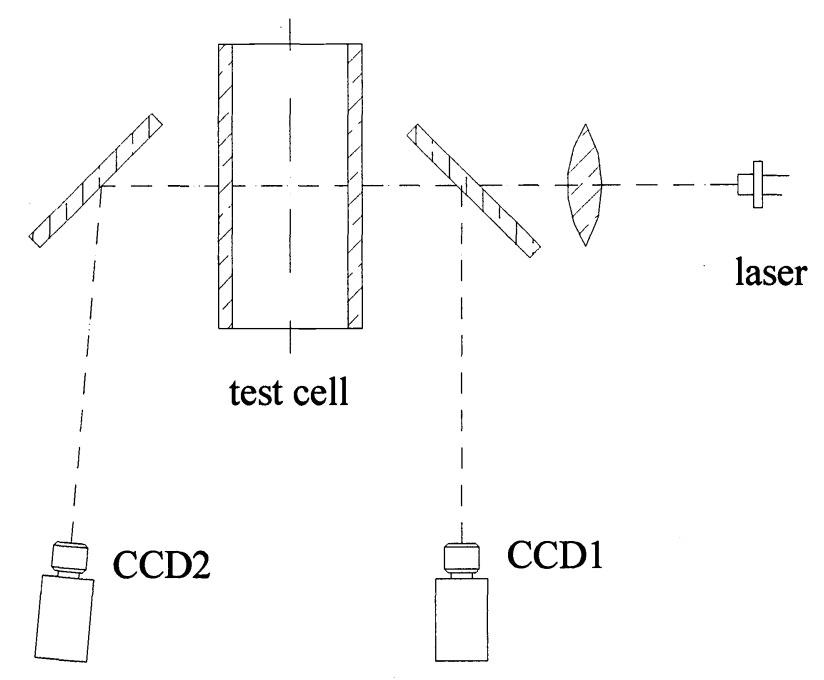

Fig. 2. Sketch of the optical system 


\section{Ground-based experiments}

We selected Fluorinert FC-75 and 5cst silicon oil as the drop and matrix liquid in space experiments respectively. However, the ground-based experiments cannot be performed in such medium because the density of drop liquid FC-75 is much higher than the matrix liquid 5cst silicon oil and then the migrations driven by the interfacial tension are greatly covered by the buoyancy driven by gravity on ground. The FC-75 is hardly to shaping as a droplet. The 5cst silicon oil and vegetable oil are selected as the drop and matrix phase liquid as ground experiment medium to replace the medium for the space experiment. Some ground-based experiments had been carried out and certain results are showed in the following figures.

In Fig3, the two interferential patterns imply the background temperature field in the test cell. The flatness and spacing density of the interferential stripes show an even temperature field and the linear degree of the temperature gradient.

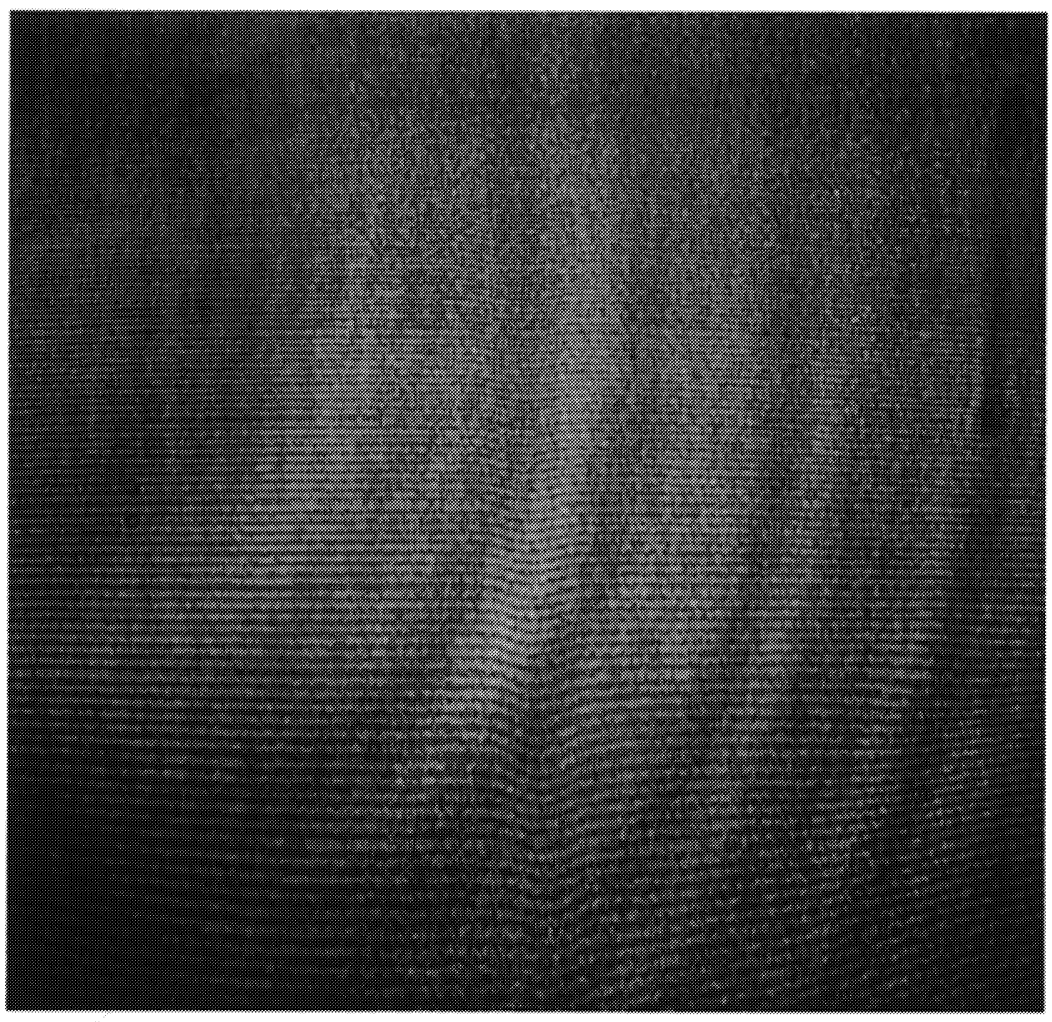

Fig. 3. Interferential fringes before drop migration

In Fig4, it shows the change of the interferential strips in the test cell when the drop is migrating to the topside. There's a trail after the migration, the reason is that there's a little solution between the drop and matrix liquid and their refractive index are not equal and the temperature of drop is different to that of the matrix liquid. The obvious changes of the interferential strips in the tail of the drop indicate the presence of the flow and the changes of the interferential strips surrounding the trail indicate the action of the interfacial tension. 


\section{Discussion}

Numerical simulations had been carried out as the pre-study of the space experiments. Two-dimensional and unsteady model of drop Marangoni migrations was calculated for the case of large Reynolds numbers and the accelerating drop migrations was determined [8]. In numerical simulation of asymmetric model, in case of large Reynolds numbers, the drop migration trajectory toward warmer region could be a non-straight line, and the migration velocity could be lower than those in YGB model. The oscillated drop trajectory could be due to the vortex in the wake of the drop tail[9]. The space experiment is designed for larger Reynolds numbers and the interferential system is expected to observing the wake and vortex.

The ground experiments showed that the space device could observe the fine structure of the drop tracks, however, it is limited in a narrow temperature range. There are two questions need to be resolved in order to improving the interferential system. The first question is to expanding the temperature range. A new interferential system is designing for continuously compensating the influence of temperature deference in relatively wider temperature range. However, it is useful only for ground experiment because it need adjusting the optical elements. Another problem is the solution of the experimental medium. The trails because of the solution maybe cover the fine structures of the wake and vortex.

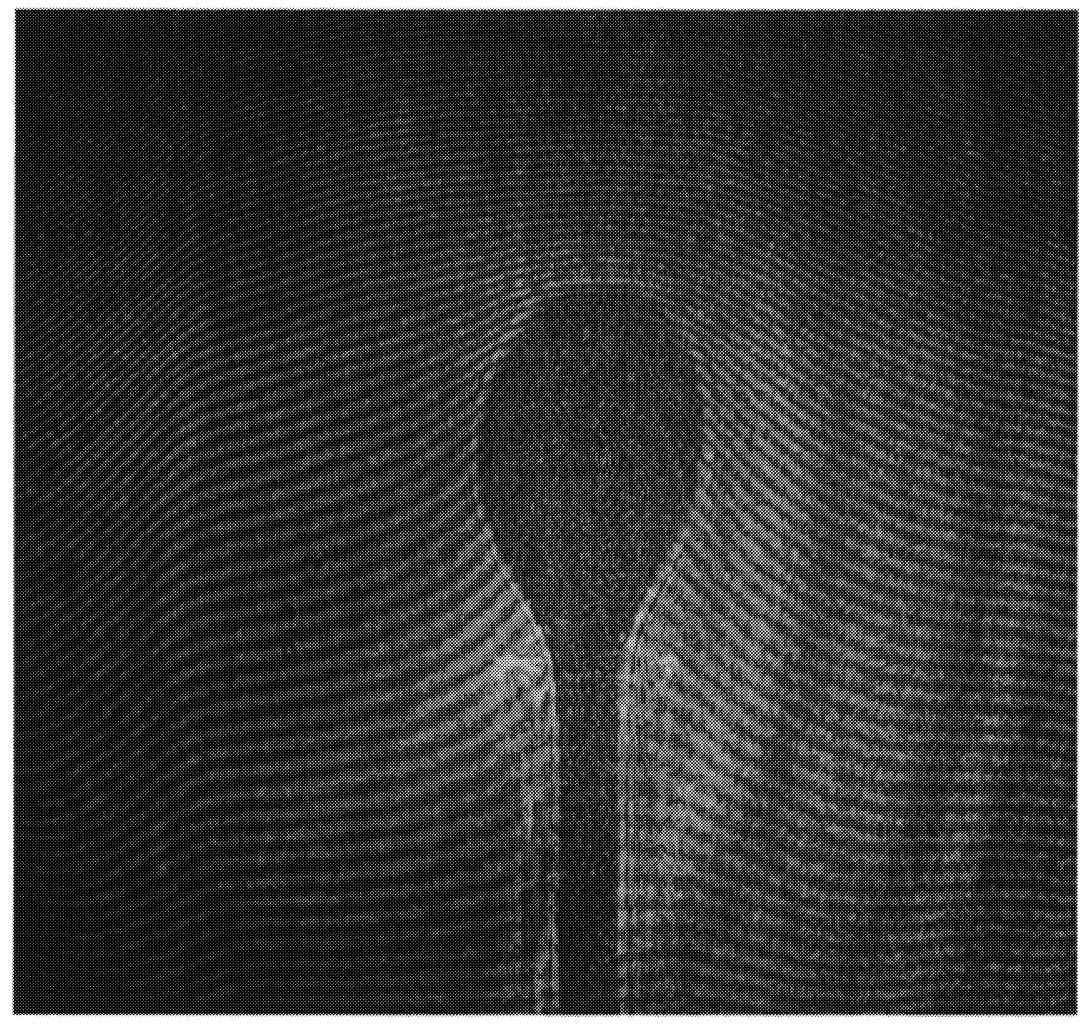

Fig.4. Interferential fringe on drop migration 


\section{References}

1. Hu, W.R., Xu, S.C., “Microgravity Fluid Mechanics" (Chinese), Beijing: Science Press, (1999).

2. Wozniak, G., Siekmann, J., Srulijes, J., “Thermocapillary bubble and drop dynamics under reduced gravity Survey and Prospects” , Z. Flugwiss Weltraumforsch, Vol 12, (1988), pp 137-147.

3. Braum, B., Ikier, C., Klein, H., "Thermocapillary migration of droplets in a binary mixture with miscibility gap during liquid/liquid phase separation under reduced gravity", J.Colloid and Interface Science, (1993), pp159-515.

4. Wozniak, G., "On the thermocapillary motion of droplets under reduced gravity", J.Colloid and Interface Science, (1991), pp141- 245.

5. Balasubraaniam, R., Lacy, C. E., Wozniak, G., et al, "Thermocapillary migration of bubbles and drops at moderate values of the Marangoni number in reduced gravity", Phys. Fluid, Vol 8, (1996), pp872.

6. Hadland, P.H., Balasubramaniam, R., Wozniak, G., et al, "Thermocapillary migration of isolated bubbles and drops at moderate to large Marangoni number and moderate Reynolds number in reduced gravity", Experiments in Fluids, Vol 26, (1999), pp240.

7. Zhang, P., Hu, L., et al. "Space experiment device on Marangoni drop migration of large Reynold numbers", Science in China (series E), Vol.44, No.6, (2001), pp605-614

8. Geng, R.H., Hu, W.R., Ao, C., "Axisymmetric drop Marangoni migration in different Reynolds numbers", IAF-98-J. 4. 06, AIAA, Australia, (1998).

9. Geng, R.H., Hu, W.R., Ao, C., "Asymmetric drop Marangoni migration of Reynolds numbers", Acta Astronautics, Vol.41, (1997), pp757. 